
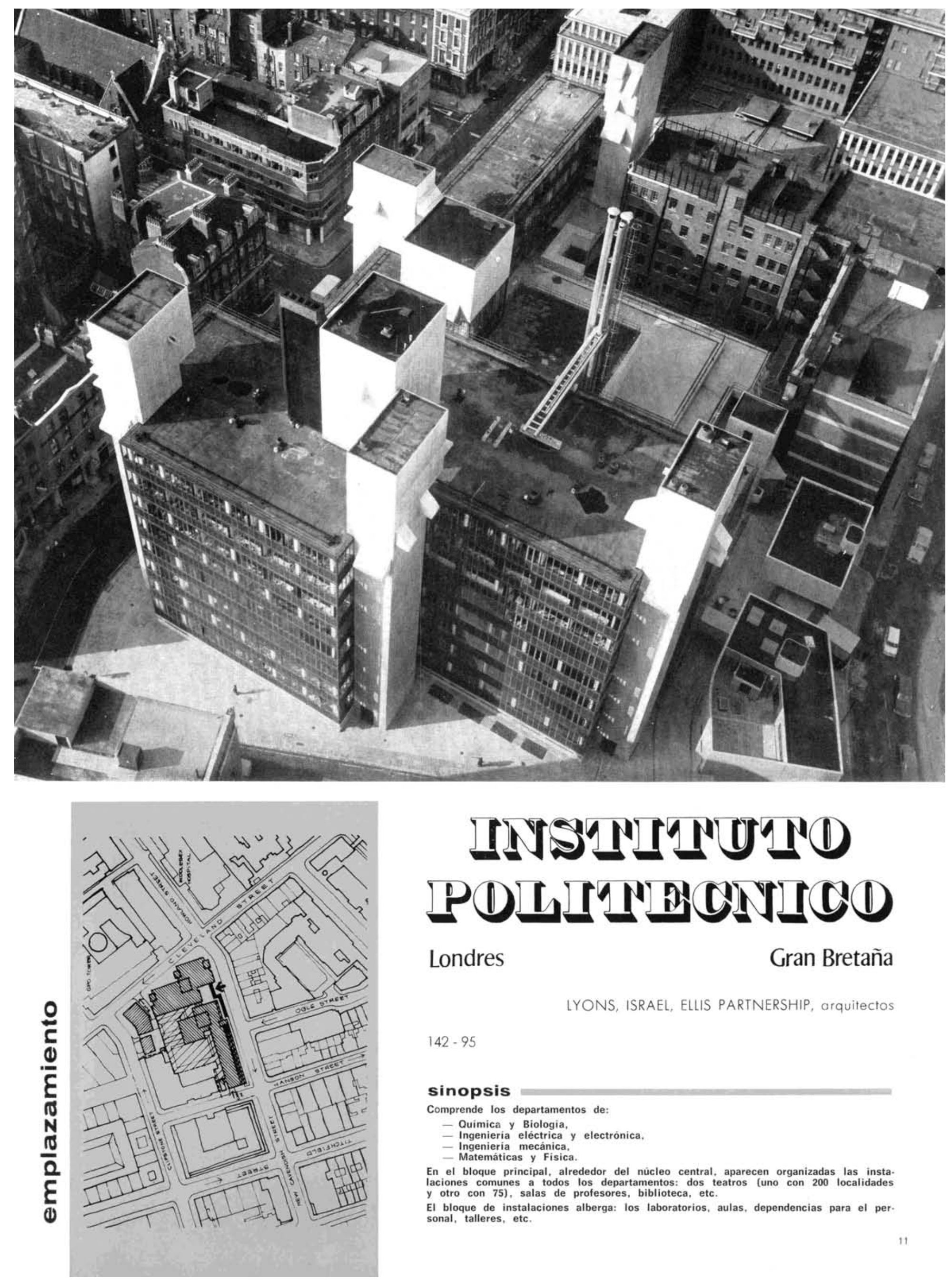

\title{
IINSTITITIT PDLITIDUINISD
}

Londres

Gran Bretaña

LYONS, ISRAEL, ELLIS PARTNERSHIP, arquitectos

$142-95$

\section{sinopsis}

Comprende los departamentos de:

- Quimica y Biologia,

- Ingenieria eléctrica y electrónica,

- Ingeniería mecánica,

En el bloque principal, alrededor del núcleo central, aparecen organizadas las insta. laciones comunes a todos los departamentos: dos teatros (uno con 200 localidades y otro con 75), salas de profesores, biblioteca, etc.

El bloque de instalaciones alberga: los laboratorios, aulas, dependencias para el personal, talleres, etc. 


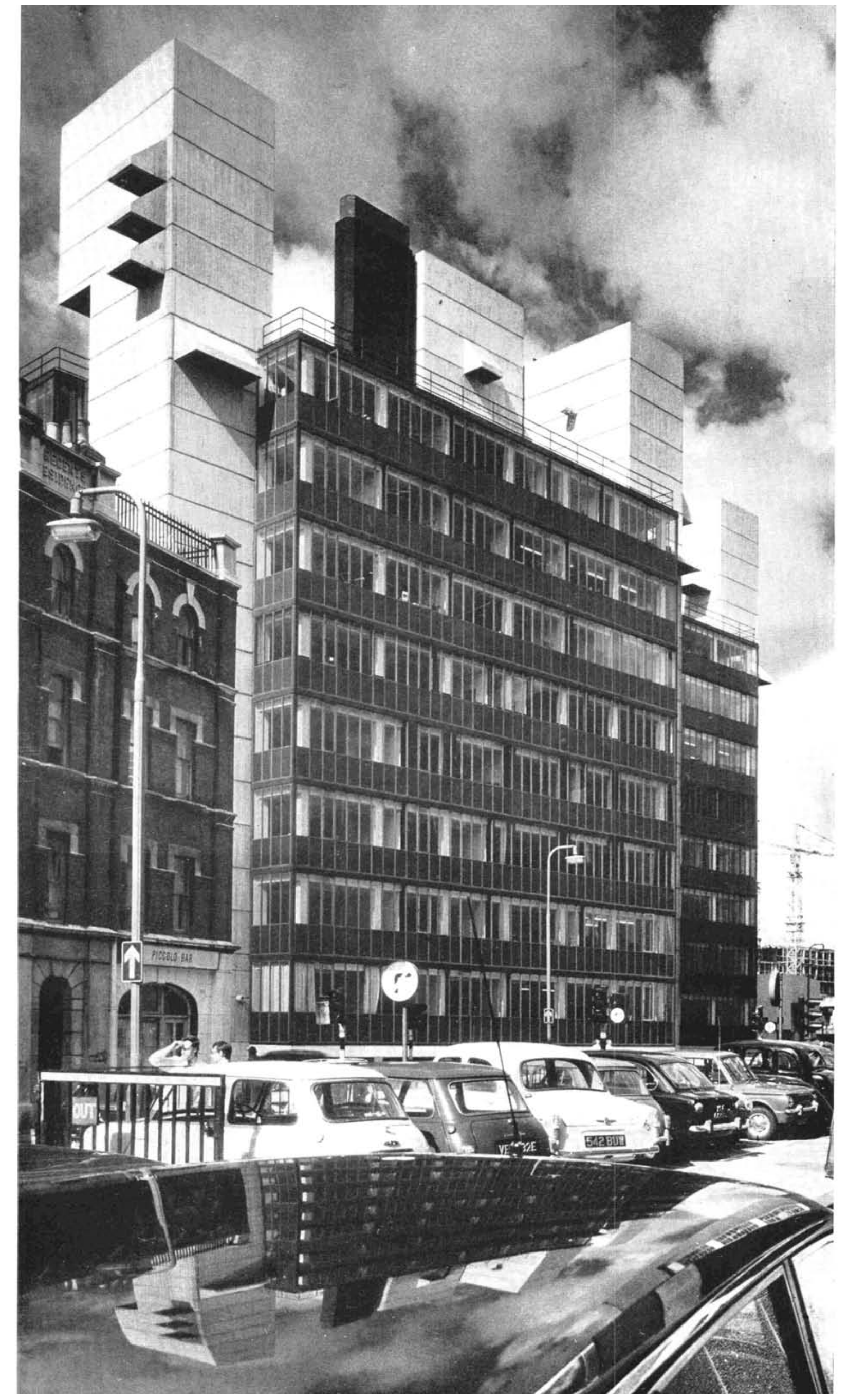


En New Cavendish Street ha sido levantado el Politécnico "Central London" (amalgama del Colegio Regent Street _fundado en 1838- y del Colegio Holborn, de Leyes, Idiomas y Comercio), que comprende los departamentos de:

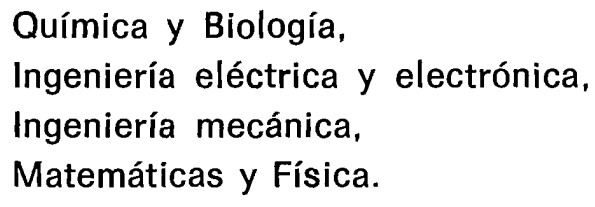

Erigido sobre un solar de unos $50.000 \mathrm{~m}^{2}$, que hace esquina a las calles Clipstone y Cleveland, satisface adecuadamente el programa de necesidades propuesto, en el que figuraba la construcción de: laboratorios, salas de dibujo, amplias naves de ingeniería, aulas, salas de conferencias, seminarios, etc.; además de los espacios necesarios para el desarrollo de la vida en común, cocinas, comedores, bar, oficinas administrativas, aparcamientos, etc. Se ha conseguido que sus instalaciones disfruten al máximo de iluminación natural, y un aprovechamiento del $80 \%$ de todas las salas, durante las horas de funcionamiento de los departamentos.

Con objeto de lograr una ordenación adecuada en un solar tan pequeño y de cumplir con las Ordenanzas municipales vigentes, los laboratorios, aulas, dependencias para el personal, talleres, etc., fueron agrupados en un bloque de 8 plantas que da a la calle Cleveland.

La entrada principal del conjunto está situada en el núcleo central. Un amplio hall conduce, por un lado, a las zonas de relación, en las que se desarrolla la vida en común: comedor, salas de estar, etc., y por otro, a la zona con las comunicaciones verticales -ascensores, escalera principal, etc.- Una serie de cortos corredores en el bloque de laboratorios permiten el acceso de los alumnos, en breve tiempo, a las comunicaciones verticales. El mencionado núcleo central asciende a través de 3 plantas, apareciendo organizadas alrede- 
dor de él las instalaciones comunes a todos los departamentos: teatros-salas de conferencias-salas de profesores, la biblioteca, etcétera.

En la planta baja, el hall de entrada y las salas comunes van acristaladas hasta el suelo (en la zona de New Cavendish, las salas comunes se abren a una terraza que domina la calle), con lo que se ha logrado establecer un contacto e integración visual entre los estudiantes y el público de la calle. Además la distribución adoptada ha reducido los obstáculos en las comunicaciones entre el personal y los estudiantes y entre los estudiantes y los diferentes departamentos.

En torno al núcleo central hay dos teatros, para diversos usos: conferencias, etc. (uno con 200 localidades y otro con 75), los cuales fueron cuidadosamente proyectados para que la audición fuese perfecta desde cualquier punto, sin necesidad del uso de micrófonos, etc., y de modo que el coloquio que siga a una conferencia sea espontáneo y libre; así, cuando un espectador situado a un tercio de la distancia total de la sala se dirija al conferenciante, se le puede oir claramente desde la última fila.

Entre las características constructivas señalaremos que las fachadas, a base de muro-cortina, fueron diseñadas sobre un módulo de 0,60 m de anchura; en su armazón, metálico, fueron utilizados perfiles laminados huecos, de bronce, y establecidas juntas de expansión a 1,82 m entre ejes; ya que la expresión de una geometría tan compleja en la forma exterior del edificio requirió un elemento unificador; y que la estructura es de hormigón armado, habiéndose utilizado elementos prefabricados pretensados en la zona de laboratorios.

Las instalaciones (mecánicas, eléctricas y sanitarias) van por encima del cielorraso, lo que permite que se puedan efectuar los trabajos de mantenimiento con un mínimo de molestias para los ocupantes de las di- 


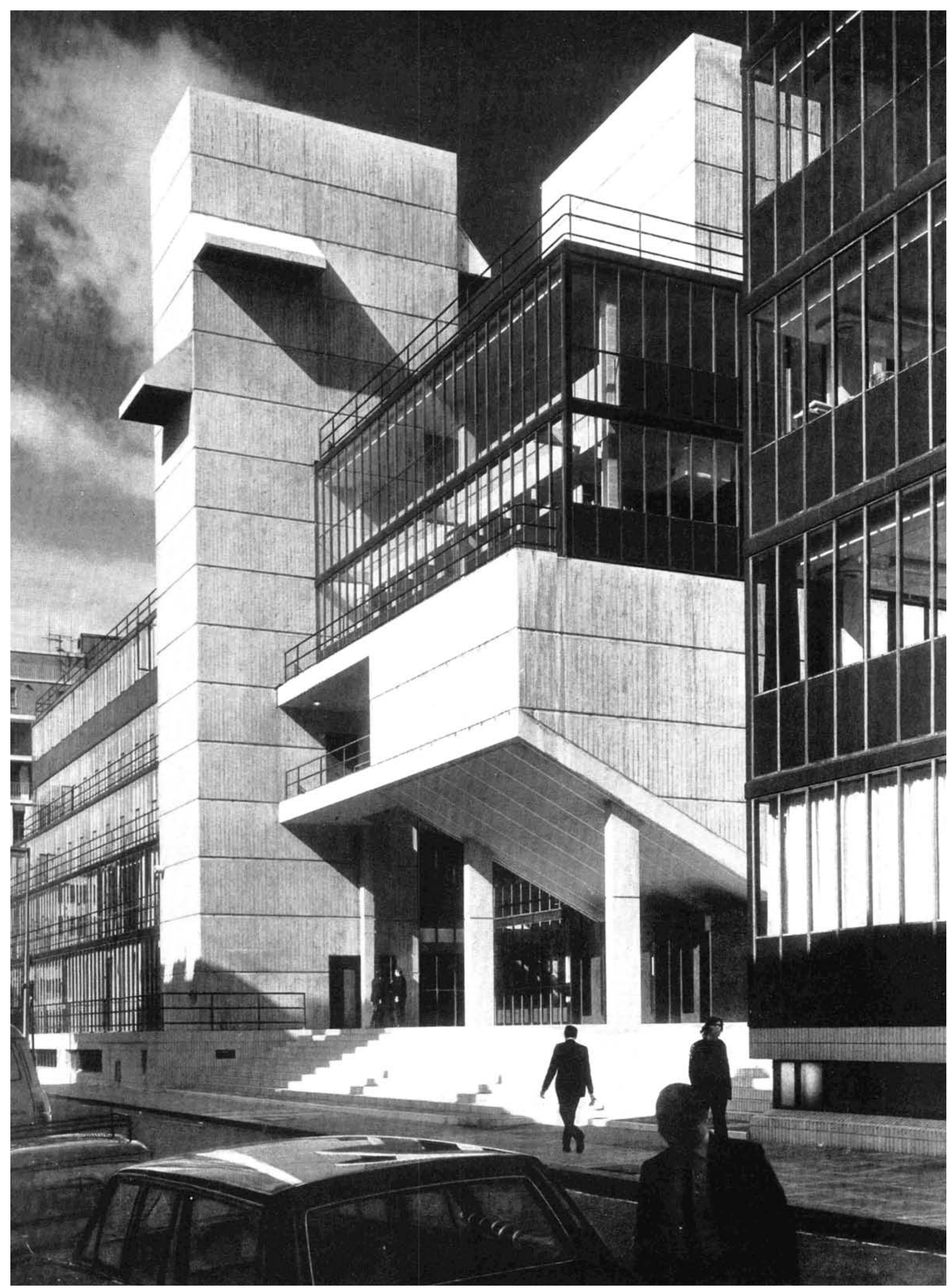




\section{plantas}

1. Montacargas.-2. Cambios.3. Paquetes. 4. Almacén de vegetales - 5. Oficio - 6 . Cámara frigorifica. - 7. Expedición. 8. Preparación de vegetales 9. Despensa. -10 . Cocina.-11 13. 13. Camarer.-14. Lavado.-15. macén - 17. Estar de empleados. 18. Bar - 19. Estar de estudiantes -20 Uso vario -21 Sala de proyectos -22 Sala de electró nica -23 . Telecomunicaciones 24 . Libros -25 . Portero -26 . Hill. 27. Oficin -28 Espera 27. Oficina. 28. Espera.-29. Reras -31 Secretaria do señ

nistración - 33. Juntas.

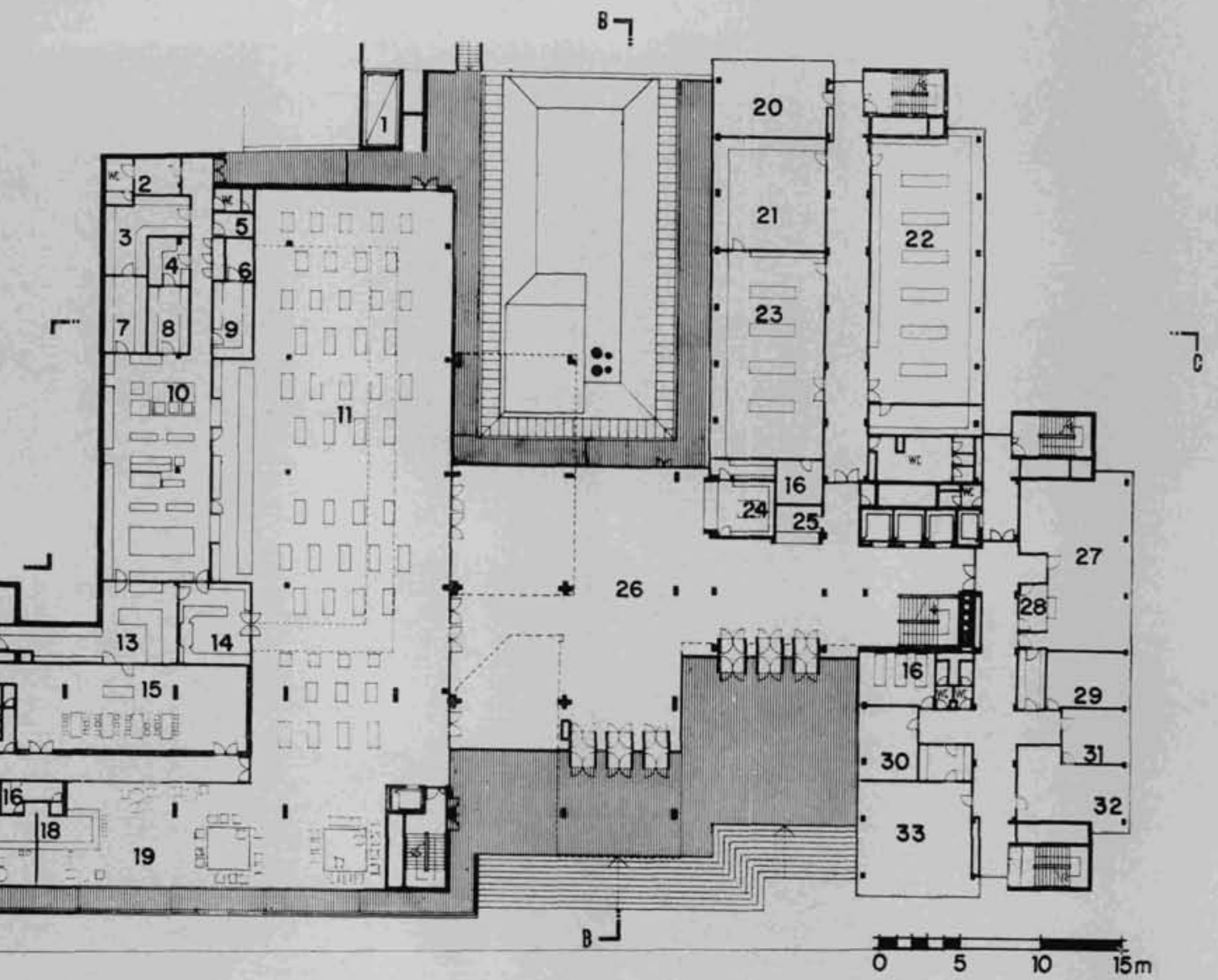

\section{baja}

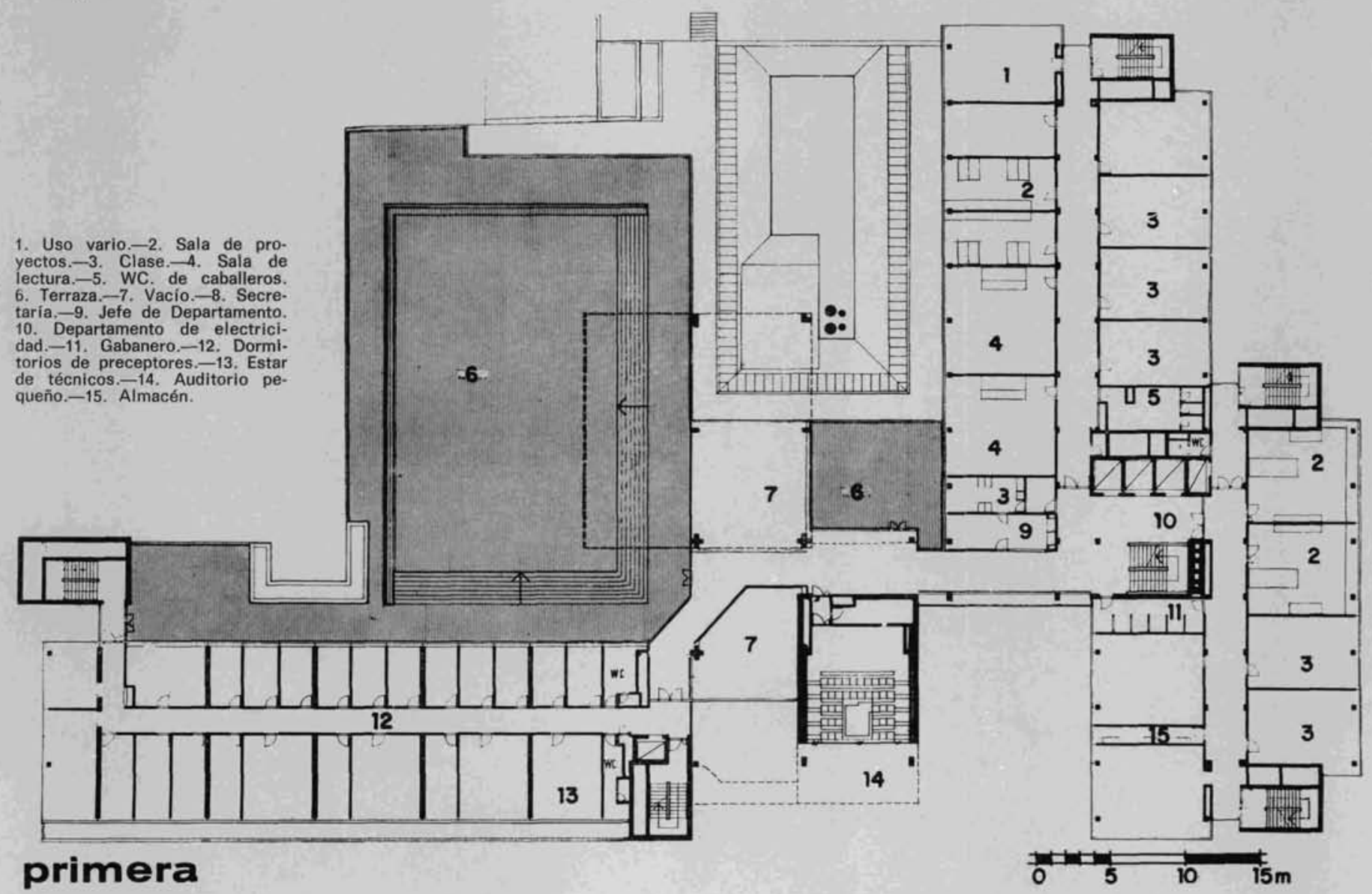



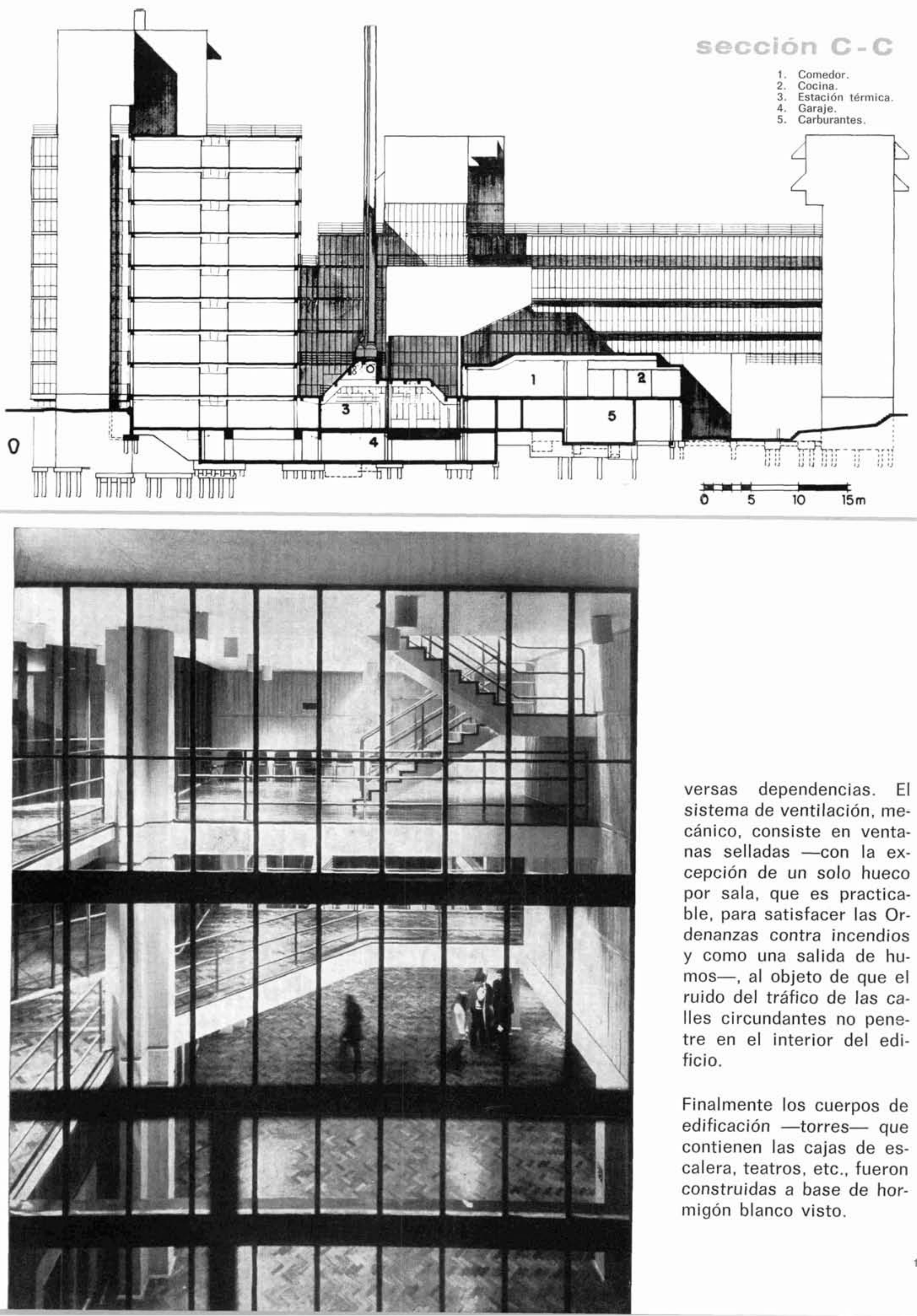

versas dependencias. El sistema de ventilación, mecánico, consiste en ventanas selladas - con la excepción de un solo hueco por sala, que es practicable, para satisfacer las Ordenanzas contra incendios y como una salida de humos-, al objeto de que el ruido del tráfico de las calles circundantes no penetre en el interior del edificio.

Finalmente los cuerpos de edificación - torres- que contienen las cajas de escalera, teatros, etc., fueron construidas a base de hormigón blanco visto. 
1. Sala de dibujo.

2. Auditorio grande

3. Secretaría.

Departamento.

. Dormitorios de preceptores.

Vacio.

Auditorio pequeño.
8. Departamento de instalaciones.

9. Gabanero

. Microscopio electrónico.

11. Proyectos de física.

13. Clase.

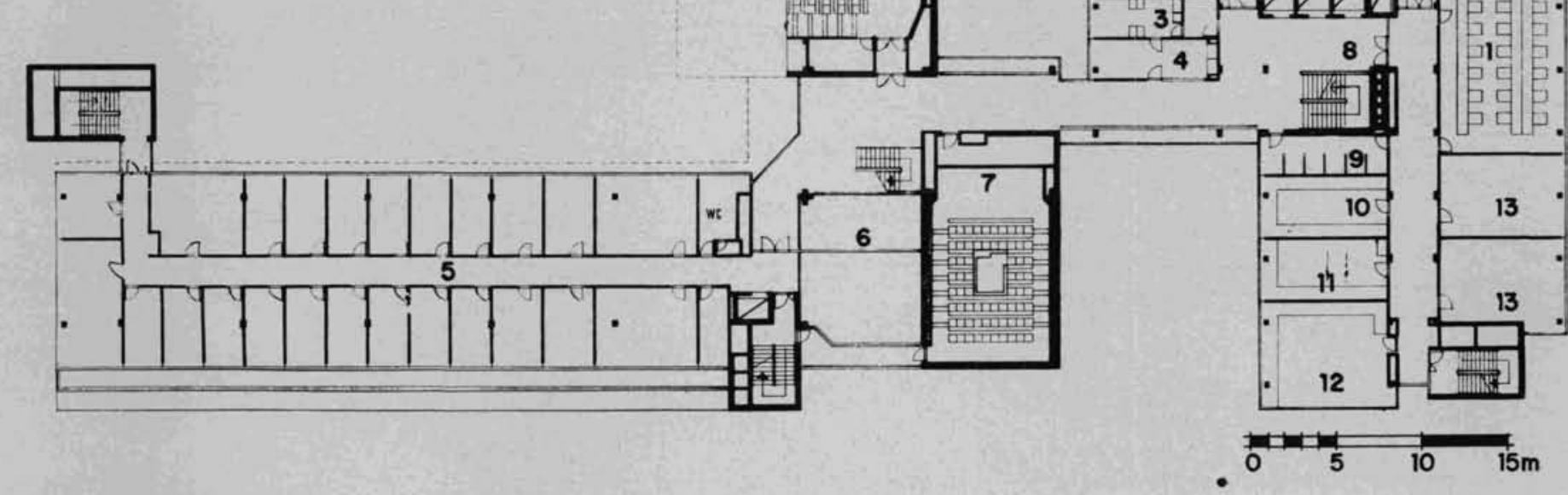

\section{segunda}

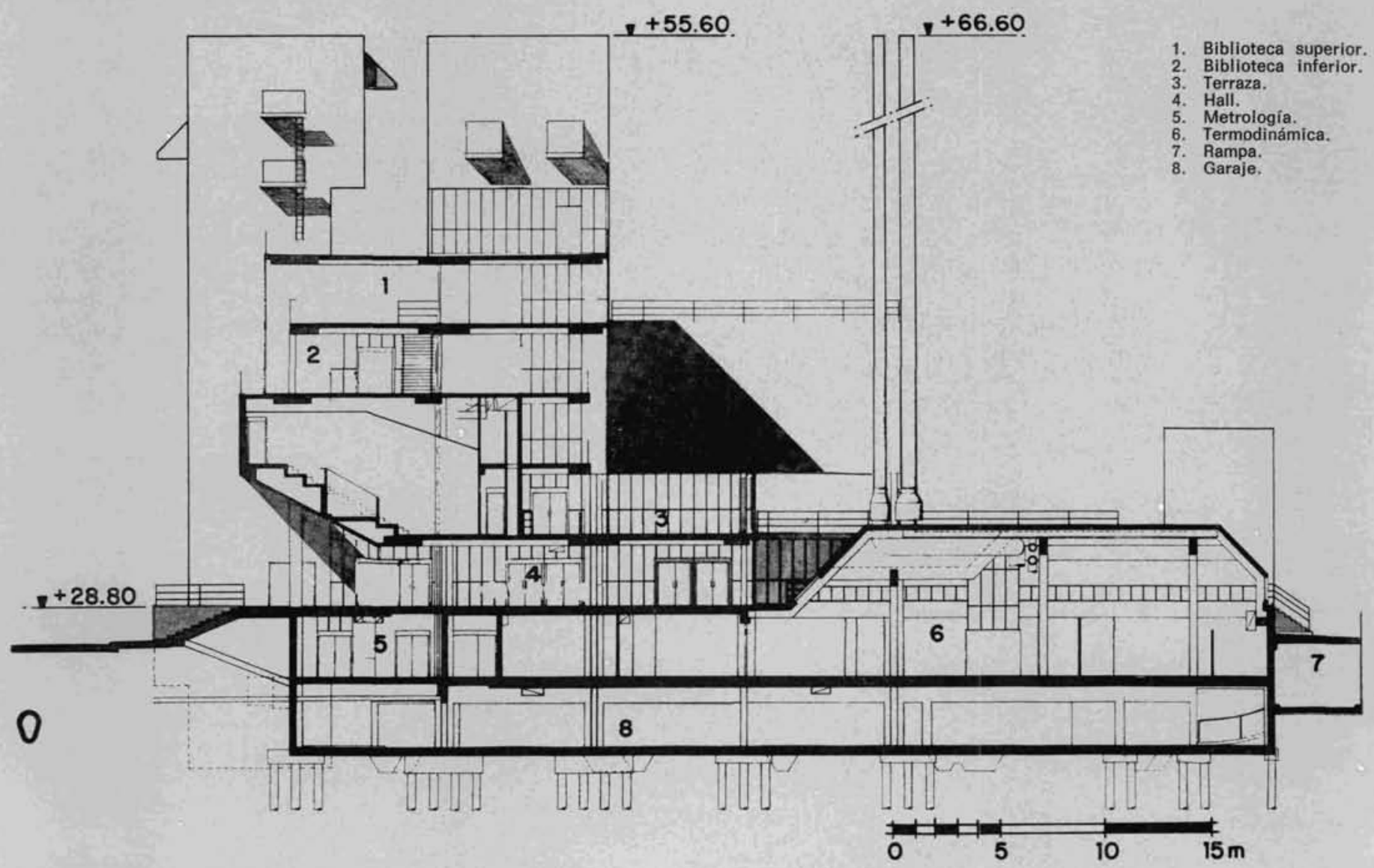

sección B-B 

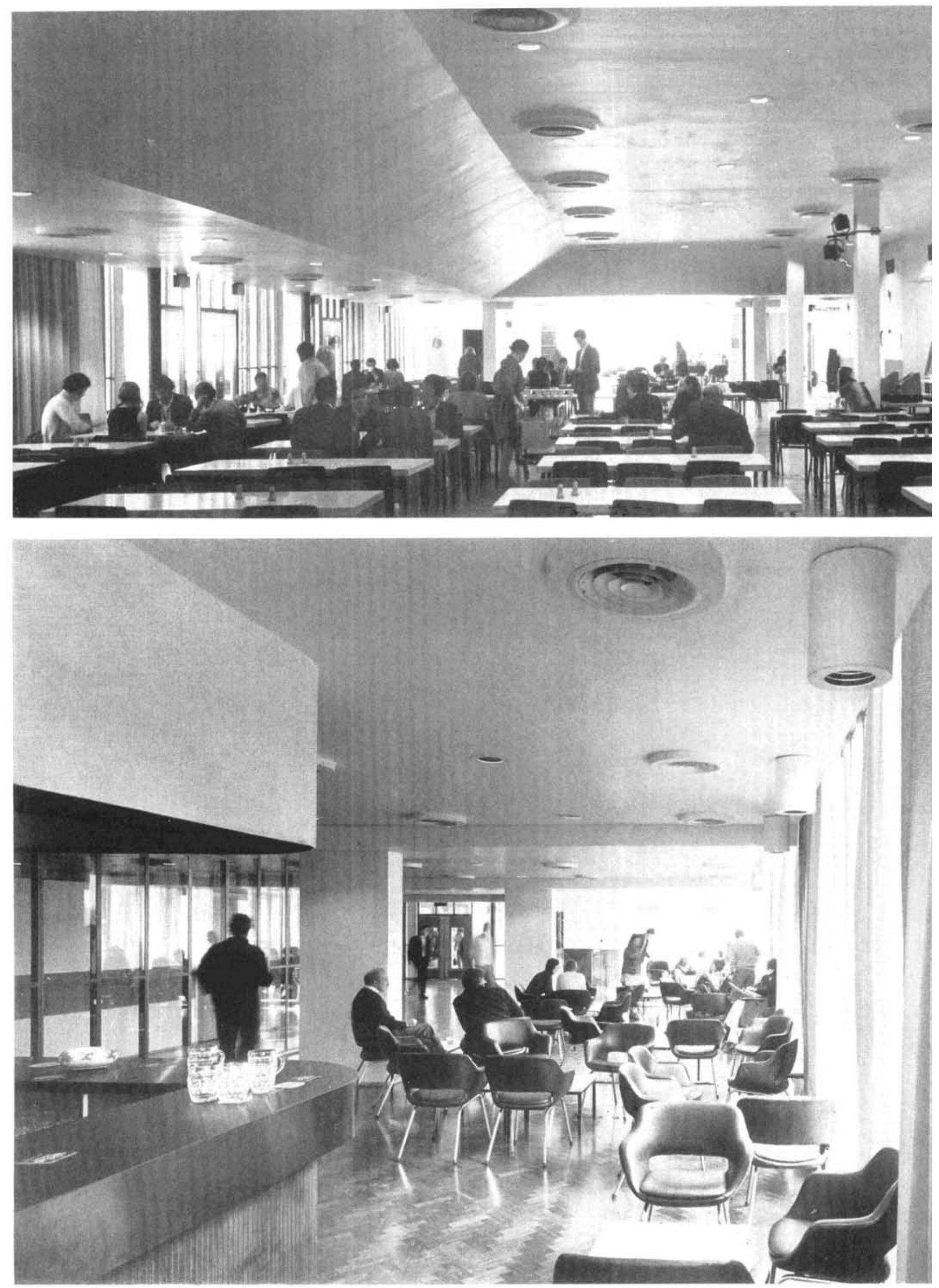


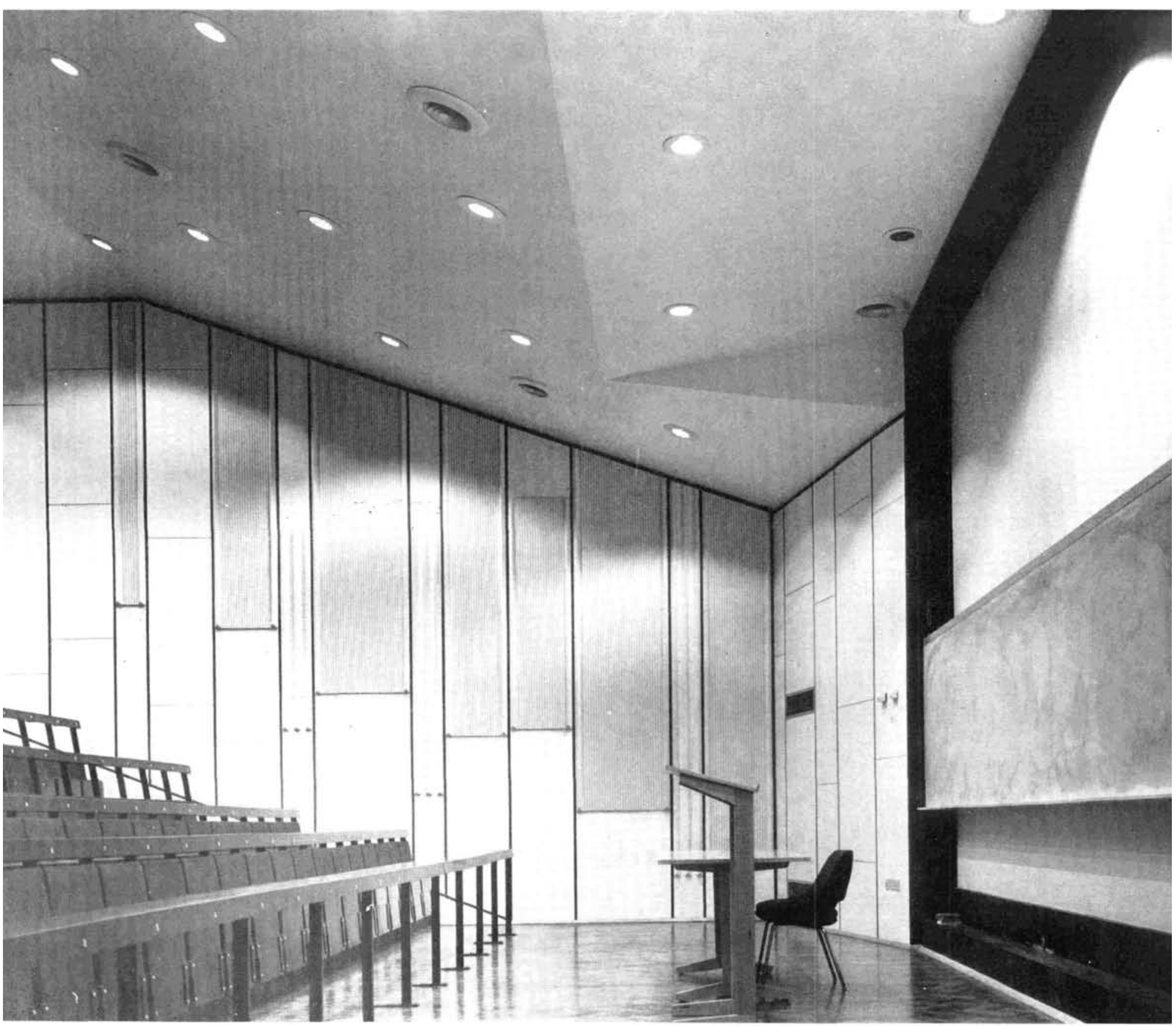

FotOS RICHARD EINZIG Y BRECHT.EINZIG, ITL

\section{résumé}

Institut polytechnique - Londres (Grande Bretagne)

Lyons, Israel, Ellis Partnership, architectes Cet Institut se compose des départements suivants:

- chimie et biologie:

- génie électrique et électronique;

- génie mécanique:

- genie mécanique;

Le bloc principal, qui entoure le noyau central, groupe les installations communes tous les departements: deux auditoriums (I'un avec 200 places assises et I'autre avec $75)$, des salles de professeurs, bibliothèque. etc.

Le bloc des installations est occupé par les laboratoires, les salles de classe, les ateliers, etc.

\section{summary}

Polytechnic Institute - London (Gt. Britain)

Lyons, Israel, Ellis Partnership, architects

This Institute has the following departments: - chemistry \& biology;

- electrical and electronic engineering:

- mechanical engineering:

- maihematics and physics.

The installations common to all the departments are arranged within the central block. around the main nucleus. These include tw 2 theatres, one holding 200 spectators and another 75 , the professors rooms and the library.

The special installations building houses the laboratories, lecture rooms, staff quarters and workshops.

\section{zusammenfassung}

Polytechnisches Institut - London (Grossbritannien)

Lyons, Israel, Ellis Partnership. Architekten

Es umfasst folgende Abteilungen:

- Chemie und Biologia;

- Elektrizitätslehre und Elektronik:

- Maschinenbau:

- Mathematik und Physik.

Im Hauptblock sind um einen zentralen Kern herum die allen Abteilungen gemeinsamen Einrichtungen angelegt: zwei Theaterràume (eines mit 200, das andere mit 75 Plätzen), Aufenthalstsräume für Professoren, Bibliothek u.s.w.

In einem anderen Block sind die Labors, Vorlesungssäle, Diensträume für das Perso nal, Werkstätten u.s.w. untergebracht. 